\title{
Gastric inflammatory fibroid polyp tumor with acute intestinal obstruction-Vanek's tumor can mimick a giant gastrointestinal stromal tumor or a gastric lymphoma
}

\author{
Francesco Fleres ${ }^{1}$, Carmelo Mazzeo ${ }^{1}$, Antonio Ieni ${ }^{2}$, Maurizio Rossitto $^{1}$, Eugenio Cucinotta ${ }^{1}$ \\ ${ }^{1}$ Department of Human Pathology of the Adult and Evolutive Age "Gaetano Barresi”, Section of General Surgery, ${ }^{2}$ Department of Human Pathology \\ of the Adult and Evolutive Age "Gaetano Barresi", Section of Anatomic Pathology, University of Messina, Messina, Italy \\ Correspondence to: Francesco Fleres, MD. Department of Human Pathology of the Adult and Evolutive Age "Gaetano Barresi”, Section of General \\ Surgery, University of Messina, Via Consolare Valeria, Messina 98125, Italy. Email: franz.fleres@gmail.com.
}

\begin{abstract}
An inflammatory fibroid polyp (IFP) is a solitary rare benign neoplasm of the gastrointestinal tract, frequently located in the gastric antrum. IFPs account for about $0.1 \%$ of all gastric polyps. We report a case of a giant gastric inflammatory polyp of $2.5 \mathrm{~cm} \times 7 \mathrm{~cm}$ that determines a gastric outlet obstruction called "ball valve syndrome" mimicking a gastrointestinal stromal tumor (GIST) and a gastric lymphoma, with an intestinal obstruction of high origin. Therefore, due to acute presentation we have decided to submit the patient to a subtotal gastrectomy. The patient was discharged two weeks later, asymptomatic. At 14 months of follow-up, patient is disease free at abdominal CT and OGDS. Depending on their size and location, IFPs can be associated with unspecific symptoms. Giant IFPs of the gastric antrum or the duodenum can determine an intermittent gastric outlet obstruction called "ball valve syndrome". Endoscopic biopsies are unhelpful and right diagnosis can be reached only with resection. In fact, only about $10 \%$ of the gastric lesions are diagnosed correctly prior to resection. Surgical treatment with complete resection with safe margins is curative. Giant IFPs are rare benign lesions whose atypical presentation can mimic GISTs, lymphomas or carcinomas. Clinical and radiological findings may not clarify the right diagnosis until histopathological evaluation aided with immunohistochemical analysis. The resection of IFPs with negative margins is curative with a good clinical outcome. In acute presentation, like in our case, surgery is the mainstay of treatment.
\end{abstract}

Keywords: Inflammatory fibroid polyp (IFP); Vanek's tumor; ball valve syndrome; gastrointestinal stromal tumor (GIST); acute gastric outlet obstruction

Received: 24 January 2018; Accepted: 04 February 2018; Published: 16 March 2018.

doi: 10.21037/jovs.2018.02.09

View this article at: http://dx.doi.org/10.21037/jovs.2018.02.09

\section{Introduction}

An inflammatory fibroid polyp (IFP) is a rare benign neoplasm of the gastrointestinal tract, frequently located in the gastric antrum. IFPs account for about $0.1 \%$ of all gastric polyps (1). The correct preoperative diagnosis is often difficult and delayed, due to IFP' rarity and to the variety and non-specificity of symptoms depending on its location and size. Thanks to the growing use of endoscopy, these lesions are increasingly identified in absence of symptoms. We report a case of a giant gastric inflammatory polyp of $2.5 \mathrm{~cm} \times 7 \mathrm{~cm}$ that determined an acute gastric outlet obstruction called "ball valve syndrome" mimicking a gastrointestinal stromal tumor (GIST) and a gastric lymphoma.

\section{Case presentation}

We report a case of a 64-year-old woman that came to our observation for recurrent episodes of abdominal colic pain in the epigastrium and in the right hypochondrium, 

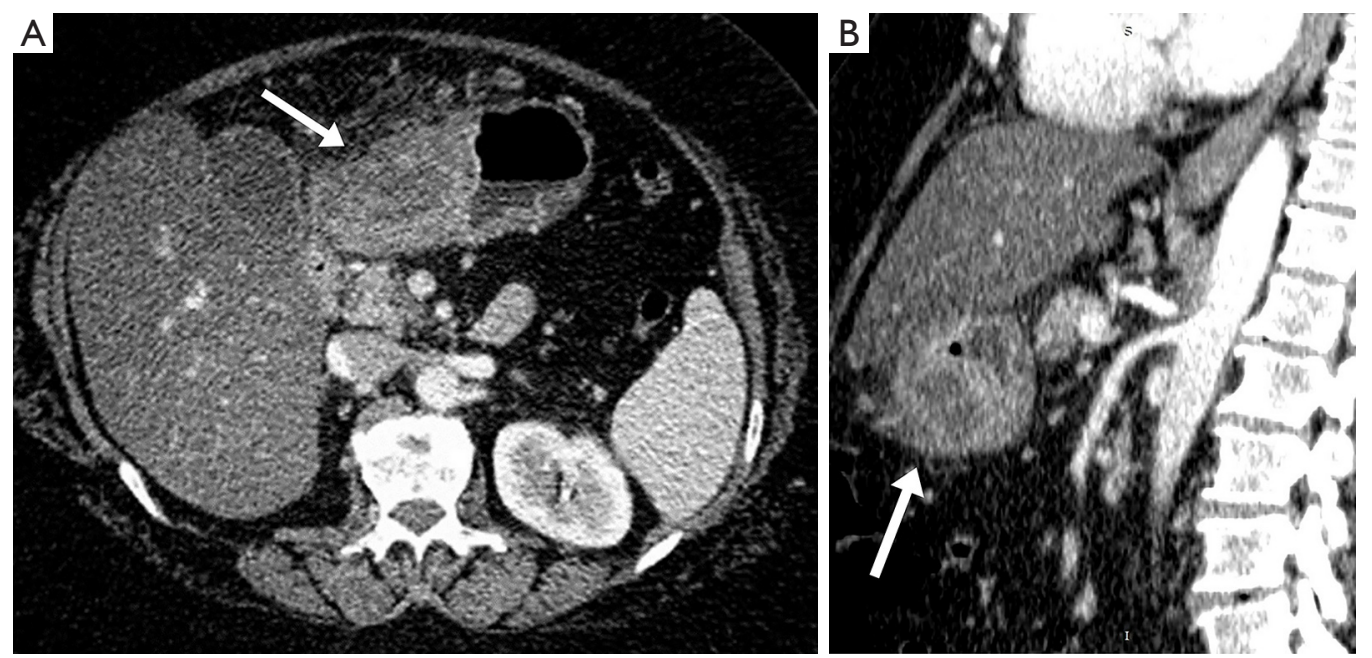

Figure 1 On transverse (A) and sagittal (B) plane, at CT imaging there was evidence the presence of a pathologic tissue between the region of the body and gastric antrum, along the greater curvature, in the anterior wall (gray arrows). This tissue presented a multiloculated aspect with an extension of $7 \mathrm{~cm} \times 5 \mathrm{~cm}$ and it appeared to grow in the context of the gastric wall resulting in marked narrowed lumen. The lesion presented markedly uneven structure, showing impregnation of its peripheral components and branches intralesional and hypodensity of the central portions as for cystic and/or necrotic-colliquative process. The lesion was developed mainly on the serous side and in the context of the omentum there were located some adenopathies globose with maximum dimensions of 14 mm. CT, computed tomography.

increasing immediately post-prandially, treated with analgesic and antispasmodic therapy with temporary advantage.

The medical history of the patient reported only arterial hypertension and severe obesity body mass index $(\mathrm{BMI})=46.8 \mathrm{~kg} / \mathrm{m}^{2}$. The laboratory data revealed a microcytic anemia (Hb. $12.1 \mathrm{gr} \%$ ) with normal value of lactate dehydrogenase ( $\mathrm{LDH})$, a mild increase of gammaglobulin, and a diagnosis of diabetes with $9.30 \%$ glycated hemoglobin level.

Tumor markers were negative: CEA $2.60 \mathrm{ng} / \mathrm{mL}$; AFP 3.20 ng/mL, CA19-9 18.50 UI/mL; CA125 94.10 U/mL; CA15-3 14.20 U/mL; CA50 38.80 U/mL; CA72-4 2.60 U/mL; Beta 2 microglobulin 2,125.00 ng/mL (range, 1,010.00-2,150.00 ng/mL); immunoglobulin IgA 4 mg/dL; IgM 337 mg/dL; IgG 1,522 mg/dL.

Therefore, the patient was submitted to an upper endoscopy [Oesophagogastroduodenoscopy (OGDS)] with evidence of a deformation of the antropyloric region similar to an ab extrinsico compression without evidence of mucosal lesions, with the impossibility of performing an endoscopic biopsy.

Abdominal computed tomography (CT) (Figure 1) confirmed the presence of pathologic tissue between the region of the body and the gastric antrum, along the greater curvature, in the anterior wall. The tissue showed a multiloculated aspect with an extension of $7 \mathrm{~cm} \times$ $5 \mathrm{~cm}$ and it appeared to grow in the context of the gastric wall resulting in marked narrowed lumen. The lesion showed markedly uneven structure, with impregnation of its peripheral components, intralesional branches and hypodensity of the central portions as for cystic and/or necrotic-colliquative process. The lesion developed mainly on the serous side and in the context of the omentum some globose adenopathies were located with maximum dimensions of $14 \mathrm{~mm}$. Moreover, a severe steatosis was present. Therefore, for this clinical picture we took into consideration the hypothesis of a GIST or a lymphoma without, however, being able to exclude other neoplastic diseases.

Within few days during the hospital stay the patient had developed an intestinal obstruction of high origin. We have submitted the patient to an endoscopic ultrasonography (EUS), which could not be performed due to the impossibility to distend the gastric lumen in correspondence of the deformation of the antro-pyloric region and without the possibility to study the gastric wall layers.

Therefore, due to acute presentation we have decided to submit the patient to an exploratory laparoscopy with extemporary biopsy of the perigastric tissue of the 
antral region. We performed a subtotal gastrectomy with lymphadenectomy and reconstruction of the digestive tract with a Roux-en-Y anastomoses and total omentectomy.

The extemporary biopsy of the perigastric tissue demonstrated only lymphoid tissue with infiltration of the adjacent adipose tissue. Even the extemporary biopsy of the falciform ligament highlighted the infiltration of the adipose tissue by lymphoid elements.

The definitive histological examination has revealed chronic inflammatory infiltration of the falciform ligament, of the gastric wall and of the greater omentum.

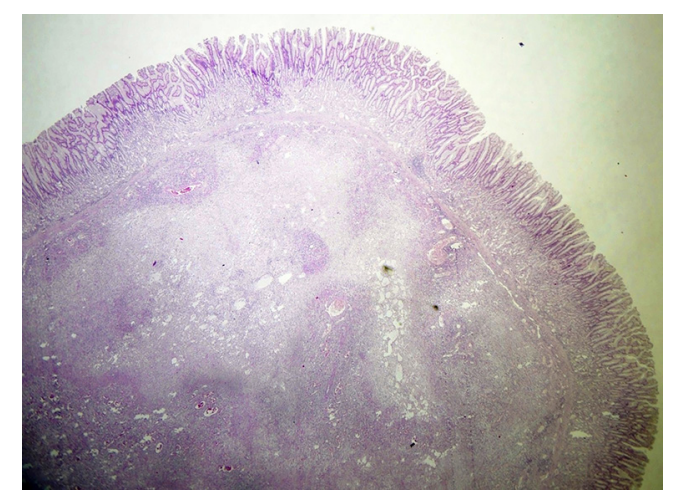

Figure 2 Polypoid appearance determined by submucosal mesenchymal proliferation, with vascular and fibroblastic components $(\mathrm{HE}, \times 80)$.
At the definitive histological examination, a neoplasm of the antrum was identified in the anterior wall measuring $2.5 \mathrm{~cm} \times 7 \mathrm{~cm}$. The neoplasm showed a diffuse inflammatory infiltration with prevalent eosinophils and polypoid intramural protrusion. The perivisceral infiltrator presented subacute character of lipophagic gigantocellular (Figures 2,3A). The features were in favor of IFP with a differential diagnosis of GIST.

Immunohistochemical staining showed positive CD34 (Figure 3B) and negative: CK AE1/AE3, Desmin, AML, S-100, CD20. Therefore, the neoplasm was compatible with the diagnosis of an IFP-tumor of Vanek. The patient was discharged 2 weeks later, asymptomatic. At 14 months of follow-up, the patient is disease-free at abdominal CT and OGDS.

\section{Discussion}

IFPs are rare submucosal growths in the gastrointestinal tract. They are benign mesenchymal gastrointestinal tumors. IFPs mainly occur in the gastric antrum and in the duodenum, but also in the small and large intestine. IFPs are solitary submucosal lesions with perivascular onion skinning and prominent eosinophilic infiltrates (1).

In 1920 Konjetzny described the first case of IFP as a "polypoid fibroma", successively in 1949 Vanek reported six cases of gastric lesions which he referred to as gastric
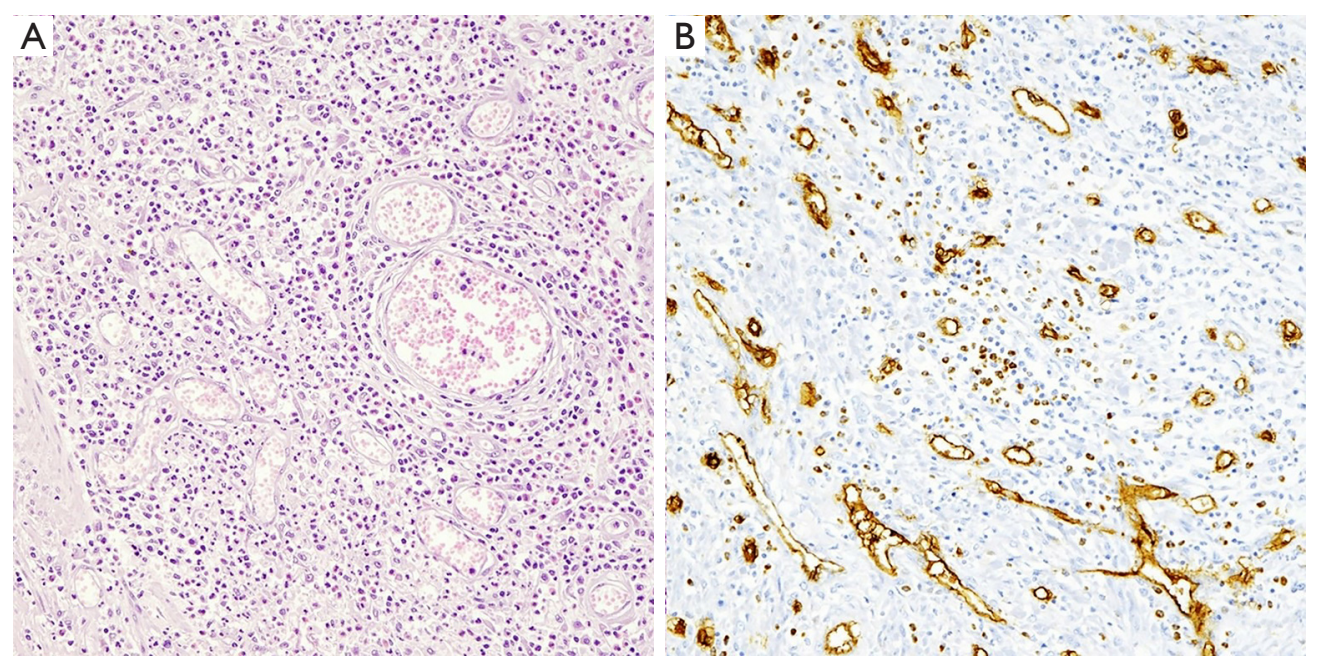

Figure 3 At higher magnification, the polyp presented mononuclear, spindle-shaped cells, arranged in whorls. An inflammatory infiltration includes blood vessels, eosinophil granulocytes, lymphocytes, macrophages and mastocytes. (A) The rich eosinophilic infiltrate is the classic one as originally described by Josef Vanek (HE, ×320); (B) CD34 showed the vascular network inside the lesion (Mayer's haemalum counterstain, $\times 320$ ). 
submucosal granuloma with eosinophilic infiltration (2). Helwig and Ranier introduced the term "inflammatory fibroid polyp" in 1953 (3).

They are usually asymptomatic, often diagnosed as incidental finding during endoscopy examinations. Depending on their size and location, IFPs can be associated with unspecific symptoms such as abdominal pain, weight loss, dyspeptic symptoms, iron deficiency anemia, intestinal obstruction, and rarely massive digestive hemorrhage (4). Giant IFPs of the gastric antrum or the duodenum can determine an intermittent gastric outlet obstruction called "ball valve syndrome" (5).

The peak age incidence occurs between 60 and 70 years (6) with a moderate male predominance. Upon the first diagnosis, the asymptomatic IFP usually measures between 2 and $5 \mathrm{~cm}$. An ileal location is most frequently responsible for intestinal intussusception (7). However, giant IFPs have been reported with a size of up to $12.5 \mathrm{~cm}$ in diameter (8).

Regarding whether the lesion is neoplastic or not, nowadays authors agreed in favor of it being a benign reactive phenomenon similar to a granuloma occurring in response to an unknown irritant agent (9). Different hypotheses have been proposed to explain the etiology of these uncommon subtype of gastric polyps: possible role of H. pylori infection, physical or metabolic factors, parasites, and allergic cause, but to date the pathogenesis of IFPs remains unclear (9-11).

Endoscopic biopsies are unhelpful and right diagnosis can be reached only with resection. In fact, only about $10 \%$ of gastric lesions are diagnosed correctly prior to resection $(10,12-14)$. Therefore, the diagnostic role of endoscopy is to identify a solitary lesion with an intramural growth.

EUS can be useful to better identify the submucosal lesion. At EUS, IFPs appear hypoechogenic and homogeneous, with indistinct margins, located within the second and third sonographic layers of the gastric wall. The numerous blood vessels within the lesion give internal echoes. EUS can support the differential diagnosis: GIST have a transmural growth with well-defined margins $(13,15,16)$.

Several differential diagnoses have to be considered. The most common benign lesions are adenomatous polyps, which are usually small. The presence of fat within the lesion characterizes intestinal lipomas at CT and magnetic resonance imaging (MRI). Lymphomas account for $20 \%$ to $40 \%$ of malignant small bowel lesions typically seen as a voluminous endoluminal tumor. GISTs have a similar appearance to IFPs but generally show partial extraluminal growth with irregular margins and a heterogeneous appearance (17).

The radiological appearance of IFPs is not specific and is scarcely reported in literature. An IFP is often described at imaging exam as an intestinal tumor growing in the lumen of the digestive tract. Balci et al. (18) have reported the MRI appearance on a T2-weighted HASTE sequence.

The peripheral enhancement is probably related to the hypervascularized nature of the IFP. On the diffusion sequence, the spherical aspect appears similar to the pathological anatomy findings: from the periphery to the center, a fleshy bud (external ring with accelerated diffusion), a fibrous ring (ring with restricted diffusion) and a central edematous and myxoid area (homogeneous diffusion).

IFPs appear either sessile or pedunculated on endoscopic examination, and may present superficial erosion/ulceration. Differential diagnosis has often to be made with GISTs, which have an incidence of $1 \%$ of all gastrointestinal tumors (19), but also with various benign mesenchymal tumors such as inflammatory pseudotumor, hemangioendothelioma, hemangiopericytoma, spindle cell carcinoid, T-cell lymphoma and solitary fibrous tumor.

Immunohistochemistry can differentiate between the two tumors, which are both positive for CD34, but only GISTs express CD117 (c-kit). Moreover, IFP is typically associated with a mutation of exon 12 of the PDGFR-A gene (20).

Wille et al. (9) have described the histologic features of IFPs, which present submucosal proliferations of spindle cells, often arranged in an onion-like pattern not only around blood vessels but sometimes also around mucosal glands. Besides proliferation of numerous capillary vessels of varying size, there were always irregularly shaped blood vessels which were often ectatic and with varying thickness of the muscular walls. Overall, there was an inflammatory reaction of varying degree, dominated by eosinophils and macrophages. Focal inflammation was seen in and around the wall of few medium-sized venous vessels. Moreover, due to the immunohistochemical similarities and since IFPs and GISTs occur exclusively in the gastrointestinal tract, Wille et al. have suggested the possibility that IFPs can be the non-neoplastic counterparts of true GIST tumors emerging from the same primitive perivascular stem cell that seems to be specific for the gastrointestinal tract.

Endoscopic polypectomy can be performed if the lesion is polypoidal and accessible. However, endoscopic resection 
may result in perforation or incomplete resection and in an increased risk of local recurrence due to its typical submucosal growth with sessile aspect. Therefore, in case of large tumors, surgical treatment with complete resection is often necessary. IFPs do not usually recur or metastasize and wedge resection with safe margins is curative.

In our case, we have had to perform a subtotal gastrectomy due to the large size of the neoplasia, which had produced an intestinal obstruction syndrome. We had not the possibility to better characterize the lesion with an EUS, first of all due to acute presentation, secondly for the impossibility to distend the gastric lumen. Therefore, we needed to resolve the obstruction syndrome and to better characterize the lesion that we thought was probably a GIST, as suggested preoperatively at the CT imaging.

\section{Conclusions}

Giant IFPs are rare benign lesions whose atypical presentation can mimic GISTs, lymphomas or carcinomas. Clinical and radiological findings may not clarify the right diagnosis until backed by histopathological evaluation through immunohistochemical analysis. The resection of IFPs with negative margins is curative with a good clinical outcome. In acute presentation, like in our case, surgery is the mainstay of treatment.

\section{Acknowledgements}

The authors thank Lo Conti Lucia that has revised the English style/spelling.

\section{Footnote}

Conflicts of Interest: The authors have no conflicts of interest to declare.

Informed Consent: Written informed consent was obtained from the patient for publication of this manuscript and any accompanying images.

\section{References}

1. Carmack SW, Genta RM, Schuler CM, et al. The current spectrum of gastric polyps: a 1-year national study of over 120,000 patients. Am J Gastroenterol 2009;104:1524-32.

2. Vanek J. Gastric submucosal granuloma with eosinophilic infiltration. Am J Pathol 1949;25:397-411.

3. Helwig EB, Ranier A. Inflammatory fibroid polyps of the stomach. Surg Gynecol Obstet 1953;96:335-67.

4. Zhang C, Cui M, Xing J, et al. Massive gastrointestinal bleeding caused by a giant gastric inflammatory fibroid polyp: A case report. Int J Surg Case Rep 2014;5:571-3.

5. Yazumi S, Nakase H, Matsushima Y, et al. The "scarfring sign" of ball valve syndrome. Gastrointest Endosc 2002;55:560.

6. Liu TC, Lin MT, Montgomery EA, et al. Inflammatory fibroid polyps of the gastrointestinal tract: spectrum of clinical, morphologic, and immunohistochemistry features. Am J Surg Pathol 2013;37:586-92.

7. Nonose R, Valenciano JS, da Silva CM, et al. Ileal Intussusception Caused by Vanek's Tumor: A Case Report. Case Rep Gastroenterol 2011;5:110-6.

8. Harned RK, Buck JL, Shekitka KM. Inflammatory Fibroid Polyps of the Gastrointestinal Tract: Radiologic Evaluation. Radiology 1992;182:863-6.

9. Wille P, Borchard F. Fibroid polyps of intestinal tract are inflammatory-reactive proliferations of CD34-positive perivascular cells. Histopathology 1998;32:498-502.

10. Albuquerque A, Rios E, Carneiro F, et al. Evaluation of clinico-pathological features and Helicobacter pylori infection in gastric inflammatory fibroid polyps. Virchows Arch 2014;465:643-7.

11. Park DY, Lauwers GY. Gastric polyps: classification and management. Arch Pathol Lab Med 2008;132:633-40.

12. Stolte $M$, Finkenzeller G. Inflammatory fibroid polyp of the stomach. Endoscopy 1990;22:203-7.

13. Sadamoto Y, Tanaka M, Harada N, et al. Gastrointestinal: gastric inflammatory fibroid polyp. J Gastroenterol Hepatol 2001;16:1069.

14. Zinkiewicz K, Zgodzinski W, Dabrowski A, et al. Recurrent inflammatory fibroid polyp of cardia: a case report. World J Gastroenterol 2004;10:767-8.

15. Chan JK. Images in pathology: myxoglobulosis of the appendix. Int J Surg Pathol 2001;9:303.

16. Matsushita M, Hajiro K, Okazaki K, et al. Gastric inflammatory fibroid polyps: endoscopic ultrasonographic analysis in comparison with the histology. Gastrointest Endosc 1997;46:53-7.

17. Bensimhon D, Soyer P, Boudiaf M, et al. Imaging of gastrointestinal stromal tumors. J Radiol 2009;90:469-80.

18. Balci NC, Radjazi S, Polat H. Adult intussusception secondary to inflammatory fibroid polyp: demonstration by MRI. Eur Radiol 2000;10:1708-10. 
19. Ridolfini MP, Cassano A, Ricci R, et al. Gastrointestinal stromal tumors. Ann Ital Chir 2011;82:97-109.

20. Daum O, Hatlova J, Mandys V, et al. Comparison of

doi: 10.21037/jovs.2018.02.09

Cite this article as: Fleres F, Mazzeo C, Ieni A, Rossitto M, Cucinotta E. Gastric inflammatory fibroid polyp tumor with acute intestinal obstruction-Vanek's tumor can mimick a giant gastrointestinal stromal tumor or a gastric lymphoma. J Vis Surg 2018;4:54. morphological, immunohistochemical, and molecular genetic features of inflammatory fibroid polyps (Vanek's tumors). Virchows Arch 2010;456:491-7. 